\title{
Tissierella praeacuta
}

National Cancer Institute

\section{Source}

National Cancer Institute. Tissierella praeacuta. NCI Thesaurus. Code C86908.

A species of obligatively anaerobic, Gram negative, rod shaped bacteria assigned to the phylum Firmicutes. This species is motile, 6 phosphogluconate dehydrogenase positive and urease and indole negative. T. praeacuta is found in the human intestinal tract and in lung abscesses and gangrenous lesions. 